\title{
A Class of Generalized Operator Quasi-Equilibrium Problems
}

\author{
Abdul Raouf ${ }^{1}$, Rajesh Kumar Gupta ${ }^{2}$, Shivani Sharma ${ }^{2}$ \\ ${ }^{1}$ Department of Mathematics, Government Degree College Mendhar, Topa, Jammu and Kashmir, India \\ ${ }^{2}$ Department of Mathematics, Lovely Professional University, Punjab, India
}

\section{Email address:}

araouf123@gmail.com (A. Raouf)

\section{To cite this article:}

Abdul Raouf, Rajesh Kumar Gupta, Shivani Sharma. A Class of Generalized Operator Quasi-Equilibrium Problems. American Journal of Applied Mathematics. Vol. 9, No. 1, 2021, pp. 16-19. doi: 10.11648/j.ajam.20210901.13

Received: January 24, 2021; Accepted: February 15, 2021; Published: March 26, 2021

\begin{abstract}
In this work, introduce and study a generalized operator quasi-equilibrium problems (in short, OQEP) in the setting of topological vector spaces. We prove some new existence results for the solution of this problem by applying $C(f)$-quasiconvex, escaping sequence in Hausdörff topological vector spaces. The results of this paper can generalize and unify previously known corresponding results of this area.
\end{abstract}

Keywords: Operator Quasi-equilibrium Problem, $C(f)$-quasiconvex, Escaping Sequence

\section{Introduction and Preliminaries}

The study of equilibrium problems was introduced by Blum and Oettle [2] in 1994. In 2005, Kazmi and Raouf [12] have intensively studied a class of operator equilibrium problems and established some existence results for solutions of this problem. In this work we establish some existence theorems for solutions of a new class of generalized operator quasiequilibrium problems (in short, OQEP). The results of this paper can be viewed as a generalization and improvement of many well-known results in the literature, see for example ([5, 11-14]).

Throughout the paper, unless otherwise specified, we use the following notations.

Let $W$ and $Z$ be Hausdörff topological vector spaces; $L(W, Z)$ be a space of all continuous linear operators from $W$ to $Z$, endowed with the topology of point-wise convergence (w.r.t.p.c.) and let $B \subset L(W, Z)$ be a nonempty convex set. Let $C: B \rightarrow \Pi(Z)$ be a multi-valued map such that for each $f \in B, C(f)$ is a solid, convex and open cone and $0 \notin C(f)$ and let $C_{0}(f)=C(f) \cup\{0\}$, where $\Pi(Z)$ denotes the family of all subsets of $Z$.

Let $Z$ be an ordered topological vector space with an ordering cone $C(f)$. Note that $C(f) \neq Z$. It is clear that the cone $C(f)$ for each $f \in B$ defines on $Z$ a partial ordering $\leq_{C(f)}$ as follows; $g \leq_{C(f)} h$ if and only if $h-g \in$
$C(f), \forall h, g \in C(f)$.

Let $S: B \rightarrow \Pi(B)$ be a multi-valued map with non-empty values. We denote by $\mathcal{F}(B)$ the family of multi-valued maps from $B \times B$ to $\Pi(Z)$. Let $F \in \mathcal{F}(B)$.

In this paper, we consider the following generalized operator quasi-equilibrium problem (OQEP). Find $f \in$ $B$ such that

$$
f \in c l_{B} S(f) \text { and } F(f, g) \not-C(f), \quad \forall g \in S(f) \text {, }
$$

where $c l_{B} S(f)$ denotes the closure of $S(f)$ in $B$.

We remark that, for suitable choices of $F, B, S, W, Z$ and $C$, OQEP (1) reduces to the problems presented in ([5, 12-14]) and the references therein. If $B \subset W$, then (1) reduces to vector equilibrium problem studied by Khaliq and Raouf [8], Khaliq [6], Khaliq and Krishan [7], Kazmi [10-11], Ansari and Yao [1] and the references therein. We omit the details.

We need the following definitions and results.

Definition 1.1. Let $P: D \subset L(W, Z) \rightarrow \Pi(Z)$ be a multivalued mapping. Then

(i) The graph of a multi-valued map $P: D \subset L(W, Z) \rightarrow$ $\Pi(Z)$ define as

$$
G(f)=\{(f, z) \in D \times Z: f \in D, \quad z \in P(f)\} .
$$

(ii) Let $P$ be a multi-valued map from range of $P$ to $D$. The 
inverse $P^{-1}$ of $P$ defined by

$$
f \in P^{-1}(z) \text { if and only if } z \in P(f) .
$$

(iii) If, for each $f \in D$ and any open set $V$ in $Z$ containing $P(f)$, there exists an open neighborhood $U$ of $f$ in $D$ such that $P(f) \subseteq V$ for all $f \in U$, then $P$ is called upper semicontinuous on $D$.

Let $B=\bigcup_{n=1}^{\infty} B_{n}$, be a subset of $L(W, Z)$ where $\left\{B_{n}\right\}_{n=1}^{\infty}$ is an increasing sequence of non-empty and compact sets such that $B_{n} \subseteq B_{n+1}$ for all $n \in N$. A sequence $\left\{f_{n}\right\}_{n=1}^{\infty}$ in $B$ is said to be escaping from $B$ (relative to $\left\{B_{n}\right\}_{n=1}^{\infty}$ ) if for each $n$ there is an $m \geq M, f_{m} \notin B_{n}$.

Let $S, T: B \rightarrow \Pi(L(W, Z))$ be multi-valued maps then the multi-valued maps $c l_{B} S, c o S, S \bigcap T: B \rightarrow \Pi(L(W, Z))$ are defined as $\left(c l_{B} S\right)(f)=c l_{B} S(f),(c o S)(f)=c o S(f)$ and $(S \bigcap T)(f)=S(f) \bigcap T(f)$, for all $f \in B$, where $\operatorname{coS}(f)$ denotes the convex hull of $S(f)$

To prove the existence of solution of OQEP(1), we shall use the following theorems which are the special cases of Theorem 2 in Ding, Kim and Tan [3] and Theorem 2 in Ding, Kim and Tan [4].

Theorem 1.1. Let $B$ be a non-empty, compact and convex subset of a Hausdörff topological vector space $E$. Suppose that $A, c_{E} A, P: B \rightarrow \Pi(B)$ are multi-valued maps such that for each $x \in B, A(x)$ is non-empty and convex set, for each $y \in B, A^{-1}(y)$ is open set in $B, c l_{E} A$ is upper semicontinuous, for each $x \in B, x \notin \operatorname{coP}(x)$ and for each $y \in B, P^{-1}(y)$ is open in $B$. Then there exists $x^{*} \in B$ such that $x^{*} \in c l_{B} A\left(x^{*}\right)$ and $A\left(x^{*}\right) \cap P\left(x^{*}\right)=\emptyset$.

Theorem 1.2. Let $B$ be a non-empty and convex subset of a locally convex Hausdörff topological vector space $E$ and $D$ be a non-empty and compact subset of $B$. Suppose that $A, P: B \rightarrow \Pi(D)$ and $c l_{E} A: B \rightarrow \Pi(B)$ be multi-valued maps such that for each $x \in B, A(x)$ is non-empty and convex set, for each $y \in B, A^{-1}(y)$ is open set in $B, c l_{E} A$ is upper semicontinuous, for each $x \in B, x \notin \operatorname{coP}(x)$ and for each $y \in D, P^{-1}(y)$ is open in $B$. Then there exists $x^{*} \in B$ such that $x^{*} \in \operatorname{cl}_{B} A\left(x^{*}\right)$ and $A\left(x^{*}\right) \cap P\left(x^{*}\right)=\emptyset$.

\section{Existence Results}

Now we give existence results for OQEP(1).

Definition 2.1. Let $C: B \rightarrow \Pi(Z)$ be a multi-valued map such that for each $f \in B, C(f)$ is a multi-valued map with convex cone values in $Z$. A multi-valued bifunction $F \in \mathcal{F}(B)$, where $\mathcal{F}(B)$ the family of multi-valued maps from $B \times B$ to $\Pi(Z)$, is called $C(f)$-quasiconvex, if for all $f, g_{1}, g_{2} \in K$ and $\lambda \in[0,1], g_{\lambda}=\lambda g_{1}+(1-\lambda) g_{2}$, we have

$$
F\left(f, g_{\lambda}\right) \subseteq F\left(f, g_{1}\right)-C(f)
$$

or

$$
F\left(f, g_{\lambda}\right) \subseteq F\left(f, g_{2}\right)-C(f) .
$$

Theorem 2.1. Let $B \subset L(W, Z)$ be a non-empty, compact and convex set. Let $C: B \rightarrow \Pi(Z)$ be a multi-valued map such that for each $f \in B, C(f)$ is a solid, convex and open cone, $0 \notin C(f)$. Let $(Z, C(f))$ be an ordered topological vector space. Let $F \in \mathcal{F}(B)$ and $S, c l_{B} S: B \rightarrow \Pi(B)$ be multi-valued maps such that for each $f \in B, S(f)$ is nonempty convex, for each $g \in B, S^{-1}(g)$ is open in $B$ and $c l_{B} S$ is upper semicontinuous. Assume that

(i) $F$ is $C(f)$-quasiconvex;

(ii) graph of $G(f)=Z \backslash(-C(f))$ is closed for all $f \in B$;

(iii) for each $g \in B, F(\cdot, g)$ is upper semi-continuous with compact values on $B$;

(iv) $F(f, f) \subseteq C_{0}(f), \forall f \in B$.

Then there exists $f_{0} \in B$ such that

$$
f_{0} \in c l_{B} S\left(f_{0}\right) \text { and } F\left(f_{0}, g\right) \nsubseteq-C\left(f_{0}\right) \forall g \in S\left(f_{0}\right) .
$$

Proof: For each $f \in B$, we define a multi-valued map $P: B \rightarrow \Pi(B)$ by

$$
P(f)=g \in B: F(f, g) \subseteq-C(f) .
$$

We show that $f \notin \operatorname{coP}(f)$, for each $f \in B$. Suppose that $f \in \operatorname{coP}(f)$, for some $f \in B$. Then there exists $f_{0} \in B$ such that $f_{0} \in \operatorname{coP}\left(f_{0}\right)$. This implies that $f_{0}$ can be expressed as

$f_{0}=\Sigma_{j \in J} \alpha_{j} g_{j}$ with $\alpha_{j} \geq 0, \Sigma_{j \in J} \alpha_{j}=1, j=1,2,3, \ldots, n$,

where $\left\{g_{j}: j \in N\right\}$ be a finite subset of $B, J \subset N$ be an arbitrary non-empty subset where $N$ denotes the set of natural numbers. This follows

$$
F\left(f_{0}, g_{j}\right) \subseteq-C\left(f_{0}\right) \text { for all } j=1,2,3, \ldots, n \text {. }
$$

Since $F$ is $C(f)$-quasiconvex, we have

$$
\begin{aligned}
F\left(f_{0}, g_{j}\right) & \subseteq F\left(f_{0}, f_{0}\right)+C\left(f_{0}\right) \\
& \subseteq C_{0}\left(f_{0}\right)+C\left(f_{0}\right) \\
& \subseteq C\left(f_{0}\right)
\end{aligned}
$$

for all $j=1,2, \ldots, n$.

$$
F\left(f_{0}, g_{j}\right) \subseteq C\left(f_{0}\right)
$$

which is a contradiction. Hence $f \notin c o P(f)$ for each $f \in B$. Now we show that $P^{-1}(g)$ is open in $B$, which is equivalent to show that $\left[P^{-1}(g)\right]^{c}=B \backslash P^{-1}(g)$ is closed.

Indeed, we have

$$
\begin{aligned}
P^{-1}(g) & =\{f \in B: g \in P(f)\} \\
& =\{f \in B: F(f, g) \subseteq-C(f)\} \\
{\left[P^{-1}(g)\right]^{c} } & =\{f \in B: F(f, g) \nsubseteq-C(f)\} .
\end{aligned}
$$

By assumptions (ii) and (iii), we claim that $\left[P^{-1}(g)\right]^{c}$ is closed in $B$, for all $g \in B$.

Indeed, let $\left\{f_{\lambda}\right\}_{\lambda \in \Gamma}$ be a net in $\left[P^{-1}(g)\right]^{c}$ such that $\left\{f_{\lambda}\right\}$ converges to $f$ (w.r.t.p.c.). Then we have $F\left(f_{\lambda}, g\right) \nsubseteq-C\left(f_{\lambda}\right)$ for each $g \in B$, that is, there exists $h_{\lambda} \in F\left(f_{\lambda}, g\right)$ such that $h_{\lambda} \notin-C\left(f_{\lambda}\right)$ or $h_{\lambda} \in G\left(f_{\lambda}\right)$ for all $\lambda \in \Gamma$. Let $A=\left\{f_{\lambda}\right\} \cup\{f\}$. Then $A$ is compact and $h_{\lambda} \in F(A, g)$ which 
is compact. Therefore $h_{\lambda}$ converges to $h$ (w.r.t.p.c.). Then, by upper semi continuity of $F(\cdot, g)$, we have $h \in F(f, g)$. Also since $G(\cdot)$ has a closed graph in $B \times Z$, we have $h \in G(f)$. Consequently, $h \in F(f, g)$ and $h \notin-C(f)$, i.e., $F(f, g) \not$ $-C(f)$. Hence $f \in\left[P^{-1}(g)\right]^{c}$ and so $\left[P^{-1}(g)\right]^{c}$ is closed in $B$ for all $g \in B$.

Thus it follows that all the hypothesis of Theorem 1.1 are satisfied. Hence there exists $f_{0} \in B$ such that

$$
f_{0} \in c l_{B} S\left(f_{0}\right) \text { and } S\left(f_{0}\right) \cap P\left(f_{0}\right)=\emptyset
$$

which implies that there exists $f_{0} \in B$ such that

$$
f_{0} \in c l_{B} S\left(f_{0}\right) \text { and } F\left(f_{0}, g\right) \not-C\left(f_{0}\right), \forall g \in S\left(f_{0}\right) \text {. }
$$

Theorem 2.2. Let $B \subset L(W, Z)$ be a non-empty subset such that $B=\bigcup_{n=1}^{\infty} B_{n}$ where $\left\{B_{n}\right\}_{n=1}^{\infty}$ is an increasing sequence of non-empty, compact and convex subsets of $B$. Let $C: B \rightarrow \Pi(Z)$ be a multi-valued map such that for each $f \in B, C(f)$ is a solid, convex and open cone, $0 \notin C(f)$. Let $(Z, C(f))$ be an ordered topological vector space. Let $F \in \mathcal{F}(B)$ and $S, c l_{B} S: B \rightarrow \Pi(B)$ be multi-valued maps such that for each $f \in B, S(f)$ is non-empty convex for each $g \in B, S^{-1}(g)$ is open in $B$ and $c l_{B} S$ is upper semicontinuous. Assume that

(i) $F$ is $C(f)$-quasiconvex;

(ii) graph of $G(f):=Z \backslash(-C(f))$ is closed for all $f \in B$;

(iii) for each $g \in B, F(., g)$ is upper semi-continuous with compact values on $B$;

(iv) $F(f, f) \subseteq C_{0}(f), \forall f \in B$;

(v) for each sequence $\left\{f_{n}\right\}_{n=1}^{\infty}$ in $B$ with $f_{n} \in B_{n}, n \in N$ which is escaping from $B$ relative to $\left\{B_{n}\right\}_{n=1}^{\infty}$, there exists $m \in N$ and $g_{m} \in B_{m} \bigcap S\left(f_{m}\right)$ such that for each $f_{m} \in \operatorname{cl}_{B} S\left(f_{m}\right)$

$$
F\left(f_{m}, g_{m}\right) \subseteq-C\left(f_{m}\right)
$$

Then there exists $f_{0} \in B$ such that

$$
f_{0} \in c l_{B} S\left(f_{0}\right) \text { and } F\left(f_{0}, g\right) \not \subset-C\left(f_{0}\right), \quad \forall g \in S\left(f_{0}\right) \text {. }
$$

Proof: Since for each $n \in N, B_{n}$ is compact and convex subset in $L(W, Z)$, applying Theorem 2.1, we have for all $n \in N$, there exists $f_{n} \in B_{n}$ such that

$$
f_{n} \in c l_{B} S\left(f_{n}\right) \text { and } F\left(f_{n}, h\right) \nsubseteq-C\left(f_{n}\right)
$$

for all $h \in S\left(f_{n}\right)$

Suppose that the sequence $\left\{f_{n}\right\}_{n=1}^{\infty}$ in $B$ be escaping from relative to $B=\bigcup_{n=1}^{\infty} B_{n}$. By assumption (iv) there exists $m \in N$ and $h_{m} \in B_{m} \bigcap S\left(f_{m}\right)$ such that for each $f_{m} \in$ $\operatorname{cl}_{B} S\left(f_{m}\right)$,

$$
F\left(f_{m}, h_{m}\right) \subseteq-C\left(f_{m}\right),
$$

which contradicts (2). Hence $\left\{f_{n}\right\}_{n=1}^{\infty}$ is not an escaping sequence from $B$ relative to $\left\{B_{n}\right\}_{n=1}^{\infty}$. Thus using the similar arguments, which have been used by Qun [15] in proving Theorem 2, there exist $r \in N$ and $f_{0} \in B_{r}$ such that $f_{n} \rightarrow f_{0}$ (w.r.t.p.c.) and $F\left(f_{0}, g\right) \subseteq G\left(f_{0}\right)$. Since $c l_{B} S: B \rightarrow \Pi(B)$ is upper semicontinuous with compact values, hence there exists $f_{0} \in B$ such that

$$
f_{0} \in c l_{B} S\left(f_{0}\right) \text { and } F\left(f_{0}, g\right) \not \subset-C\left(f_{0}\right), \forall g \in S\left(f_{0}\right) .
$$

Theorem 2.3. Let $B$ be a non-empty for each convex subset of a locally convex Hausdorff topological vector space $L(W, Z)$ and $D$ be a nonempty compact subset of $B$. Let $C: B \rightarrow \Pi(Z)$ be a multi-valued map such that for each $f \in B, C(f)$ is a solid, convex and open cone, $0 \notin C(f)$. Let $(Z, C(f))$ be an ordered Hausdorff topological vector space. Let $F \in \mathcal{F}(B)$ and $S, \operatorname{cl}_{B} S: B \rightarrow \Pi(B)$ be multi-valued maps such that for each $f \in B, S(f)$ is non-empty convex, for each $g \in B, S^{-1}(g)$ is open in $B$ and $c l_{B} S$ is upper semicontinuous. Assume that

(i) $F$ is $C(f)$-quasiconvex;

(ii) The graph of $G(f):=Z \backslash(-C(f))$ is closed for all $f \in B$;

(iii) for each $g \in B, F(\cdot, g)$ is upper semi-continuous with compact values on $B$;

(iv) $F(f, f) \subseteq C_{0}(f), \forall f \in B$.

Then there exists $f_{0} \in B$ such that

$$
f_{0} \in c l_{B} S\left(f_{0}\right) \text { and } F\left(f_{0}, g\right) \not \subset-C\left(f_{0}\right), \quad \forall g \in S\left(f_{0}\right) \text {. }
$$

Proof: Let $P: B \rightarrow \Pi(B)$ be a multi-valued map define by

$$
P(f):=\{g \in D: F(f, g) \subseteq-C(f)\} \forall f \in B .
$$

Then by using the same argument, which we have used in proving Theorem 2.1, we have $f \notin B$ for each $f \in B$ and $P^{-1}(g)$ is open for each $g \in D$. Thus all the conditions of Theorem 1.2 are satisfied. Hence there exists $f_{0} \in B$ such that

$$
f_{0} \in c l_{B} S\left(f_{0}\right) \text { and } S\left(f_{0}\right) \cap P\left(f_{0}\right)=\emptyset
$$

which implies that there exists $f_{0} \in B$ such that

$$
f_{0} \in c l_{B} S\left(f_{0}\right) \text { and } F\left(f_{0}, g\right) \not \subset-C\left(f_{0}\right), \forall g \in S\left(f_{0}\right) \text {. }
$$

\section{Application}

As an immediate application of theorems proved in Section 2, we obtain as special cases, the following existence results for the solutions of generalised operator quasivariational-like inequality problem (in short, GOQVLIP) and generalized operator variational-like inequality problem (in short, GOVLIP).

Theorem 3.1. Let $W, Y$ and $Z$ be three Hausdörff topological vector spaces and $B$ be a non-empty, compact and convex subset of $L(W, Z)$. Let $(Y, C(f))$ be an ordered Hausdörff topological vector space. Let $M: B \times Z \rightarrow$ $\Pi(L(W, Y))$ be a multi-valued map; $\eta: B \times B \rightarrow L(W, Y)$ be bifunction; $T: B \rightarrow \Pi(Z)$ and $S: B \rightarrow \Pi(B)$ be multivalued maps. Define

$$
F(f, g)=\operatorname{Max}\langle M(f, \nu), \eta(g, f)\rangle
$$


for all $f, g \in B$, where $\nu \in T(f)$ be assume that all the other conditions of Theorem 2.1 holds. Then the GOQVLIP of finding $f_{0} \in B$ such that for each $g \in S\left(f_{0}\right)$ there exists $\nu_{0} \in T\left(f_{0}\right)$ satisfying

$$
f_{0} \in c l_{B} S\left(f_{0}\right) \text { and } \operatorname{Max}\left\langle M\left(f_{0}, \nu_{0}\right), \eta\left(g, f_{0}\right)\right\rangle \nsubseteq-C\left(f_{0}\right) \text {, }
$$

has a solution.

Corollary 3.1. If $S(f)=B$ for all $f \in B$, in Theorem 3.1. Then the GOVLIP of finding $f_{0} \in B$ such that for each $g \in B$, there exists $\nu_{0} \in T\left(f_{0}\right)$ satisfying

$$
f_{0} \in B \text { and } \operatorname{Max}\left\langle M\left(f_{0}, \nu_{0}\right), \eta\left(g, f_{0}\right)\right\rangle \nsubseteq-C\left(f_{0}\right),
$$

has a solution.

Remark 3.1. If $B \subset W$ in Corollary 3.1, then we obtain the existence results of compact and non-compact settings of [9].

\section{Conclusion}

In this work, we studied a new class which is known as a generalized operator quasi-equilibrium problem and establish existance results for using escaping sequence and $C(f)$-qusasiconvex, in the setting of topological vector spaces. The operator quasi-equilibrium is a generalization and improvement, include not only scalar and vector equilibrium and variational inequalities problems as special cases, but have sufficient evidence for their importance to study, see ([5, 12])

\section{Acknowledgements}

The authors sincerely thank the reviewers for their valuable suggestions and useful comments that have led to the present improved version of the original manuscript.

\section{References}

[1] Ansari, Q. H. and Yao, J. C., An existence result for the generalized vector equilibrium problem, Appl. Math. Letters 12 (1999), 53-56.

[2] Blum, E. and Oettli, W., From optimization and variational inequalities to equilibrium problems, Math. Stud. 63 (1994), 123-145.
[3] Ding, X. P., Kim, W. K. and Tan, K. K., Equilibria of non-compact generalized game with $L^{*}$-majerized preferences, J. Math. Anal. Appl. 164 (1992), 508-517.

[4] Ding, X. P., Kim, W. K. and Tan, K. K., Equilibria of non-compact generalized game with L-majerized correspendences, International J. Math. \& Math. Sci. 17 (1994), 783-790.

[5] Domokos, A and Kolumbán, J., Variational inequalities with operator solutions, J. Global. Optim. 23 (2002), 99110.

[6] Khaliq, A., On generalized vector equilibrium problems, Gaint 19 (1999), 69-83.

[7] Khaliq, A. and Krishan, S., Vector quasi-equilibrium problems Bull. Austral. Math. Soc., 68 (2003), 295-302.

[8] Khaliq, A. and Raouf, A., Geeneralized vector quasiequilibrium problems, Adv. Nonl. Vari. Ineq. 7(1)(2004), 47-57.

[9] Khaliq, A. and Raouf, A., Existence of solutions for generalized vector variational-like inequalities, South East Asian J. Math. \& Math. Sc. 2 (1) (2003), 1-14.

[10] Kazmi, K. R., A variational principle for vector equilibrium problems, Proc. Indian Acad. Sci. (Math. Sci), 111 (2001), 465-470.

[11] Kazmi, K. R., On vector equilibrium problem, Proc. Indian Acad. Sci., 110 (2000), 213-223.

[12] Kazmi, K. R. and Raouf, A., A class of operator equilibrium problem, J. Math. Annl. and Appl. 308 (2005), 554-564.

[13] Kazmi, K. R. and Raouf, A., Preturbed Operator Equilibrium Problems South East Asian J. Math. \& Math. Sc. 8 (1) (2009), 91-100.

[14] Kim, J. K. and Raouf, A. , A Class of Generalized Operator Equilibrium Problems Filomat 31: 1 (2017) 18.

[15] Qun, L., Generalized vector variational-like inequalities, In: Vector Variational Inequalities and Vector Equilibria, pp. 363-369, Nonconvex Optim. Appl. Vol. 38, Kluwer Acad. Publ. Dordrecht, 2000. 